\title{
Digital Signature
}

National Cancer Institute

\section{Source}

National Cancer Institute. Digital Signature. NCI Thesaurus. Code C80447.

Extra data appended to a message which identifies and authenticates the sender and message data using public-key encryption. 